\title{
artigo
}

\section{Tratamento Tópico para o Sangramento em Feridas Tumorais: Uma Revisão Integrativa}

\author{
Topical Treatment for Bleeding from Tumor Wounds: An Integrative Review
}

Tratamiento tópico para el sangrado de heridas tumorales: una revisión integral

\section{RESUMO}

Objetivo: realizar uma revisão integrativa da literatura, focada na identificação e avaliação de estudos primários objetivando o controle tópico do sangramento em feridas tumorais. Métodos: foi feita pesquisa bibliográfica no período de dezembro de 2020 a janeiro de 2021 nas bases de dados Scopus, Cinahl, PubMed e Web of Science e usados os descritores wounds and injuries, hemorrhage, neoplasms e nursing e as palavras chaves symptom management, bleeding e therapeutics. Resultados: A amostra final foi composta por 06 estudos. Os delineamentos mais frequentes foram série de casos/relato de caso, com cinco estudos $(83,3 \%)$, e um estudo de coorte $(16,7 \%)$. Portanto, cinco estudos (83,3\%) apresentaram nível de evidência 6 (fraca) e um $(16,7 \%)$, nivel de evidência 4 (moderada). Conclusão: recomenda-se que estudos posteriores com melhor delineamento metodológico sejam conduzidos a fim de produzir evidências mais concretas.

DESCRITORES: Sangramento; Oncologia; Feridas; Cuidados Paliativos.

\section{ABSTRACT}

Objective: to carry out an integrative literature review, focused on the identification and evaluation of primary studies aiming at the topical control of bleeding in tumor wounds. Methods: bibliographic research was carried out from December 2020 to January 2021 in the Scopus, Cinahl, PubMed and Web of Science databases and used the keywords wounds and injuries, hemorrhage, neoplasms and nursing and the keywords symptom management, bleeding and therapeutics. Results: The final sample consisted of 06 studies. The most frequent designs were case series / case report, with five studies (83.3\%), and one cohort study $(16.7 \%)$. Therefore, five studies (83.3\%) presented evidence level 6 (weak) and one (16.7\%), evidence level 4 (moderate). Conclusion: it is recommended that further studies with a better methodological design be conducted in order to produce more concrete evidence.

DESCRIPTORS: Bleeding; Oncology; Wounds; Palliative care.

\section{RESUMEN}

Objetivo: realizar una revisión integradora de la literatura, enfocada a la identificación y evaluación de estudios primarios orientados al control tópico del sangrado en heridas tumorales. Métodos: se realizó una investigación bibliográfica de diciembre de 2020 a enero de 2021 en las bases de datos Scopus, Cinahl, PubMed y Web of Science y se utilizaron las palabras clave heridas y lesiones, hemorragia, neoplasias y enfermería y las palabras clave manejo de síntomas, sangrado y terapéutica. Resultados: La muestra final constó de 06 estudios. Los diseños más frecuentes fueron series de casos / relatos de casos, con cinco estudios $(83,3 \%)$ y un estudio de cohortes (16,7\%). Por tanto, cinco estudios $(83,3 \%)$ presentaron nivel de evidencia 6 (débil) y uno $(16,7 \%)$, nivel de evidencia 4 (moderado). Conclusión: se recomienda que se realicen más estudios con un mejor diseño metodológico para producir evidencia más concreta.

DESCRIPTORES: Sangrado; Oncología; Heridas; Cuidados paliativos.

RECEBIDO EM: 31/01/2021 APROVADO EM: 15/02/2021

\section{Thalyta Cássia de Freitas Martins}

Doutoranda em Saúde Pública. Graduada em Enfermagem pela Universidade Federal de Viçosa. Mestre em Ciências da Saúde pela Universidade Federal de Viçosa (UFV). Especialista em Enfermagem Oncológica pelo Programa de Residência Multiprofissional do Instituto Nacional de Câncer (INCA). Especialista em Estomaterapia pela Universidade Federal do Rio de Janeiro (UERJ). Atualmente é doutoranda do Programa de Saúde Pública na Fundação Oswaldo Cruz (FioCruz).

ORCID: 0000-0002-6225-7245 


\section{Poliana Miranda}

Mestre em Ciências da Saúde. Enfermeira graduada pela Universidade Federal dos Vales do Jequitinhonha e Mucuri - UFVJM. Mestre em Ciências da Saúde pela Universidade Federal de Viçosa - UFV. Residente em Enfermagem em Terapia Intensiva Oncológica no Hospital do Câncer de Barretos (HCB - Hospital de Amor).

ORCID: 0000-0001-5864-6961

\section{Samanta Cristina de Almeida Pereira}

Graduação em Enfermagem. Graduada em Enfermagem pelo Centro Universitário Barão de Mauá. Residente em Oncologia no Hospital do Câncer de Barretos (HCB - Hospital de Amor).

ORCID: 0000-0001-7104-5300

\section{Matheus Gomes de Souza}

Graduação em Enfermagem. Graduação em Enfermagem. Residente em Enfermagem em Terapia Intensiva Oncológica no Hospital do Câncer de Barretos (HCB - Hospital de Amor).

ORCID: 0000-0001-8207-6997

\section{INTRODUÇÃO}

A $s$ Feridas Tumorais se originam a partir da infiltração de células neoplásicas nas estruturas da pele, levando ao rompimento da integridade tissular e causando desordens vasculares que geram a necrose tecidual ${ }^{1}$. O processo de carcinogênese, caracterizado por uma proliferação celular descontrolada, leva ao surgimento das lesões ulcerativas ${ }^{2}$. A literatura mostra que $5 \%$ a $10 \%$ dos pacientes com câncer avançado são acometidos por essas lesões ${ }^{3,4}$ e dentre seus principais sintomas, destacam-se a dor, o odor fétido, a exsudação intensa e a friabilidade ${ }^{5,6}$. Dentre estes, o sangramento é descrito pela literatura como um sintoma pouco estudado devido ao fato de ser imprevisível e apresentar intensidade diversificada ${ }^{7}$.

As feridas tumorais são fonte de grande sofrimento psicológico e social por gerarem uma grande alteração da imagem corporal dos pacientes, sobretudo devido a seus sintomas físicos como o odor, a dor, o prurido, o exsudato excessivo e sangramento. Os pacientes relatam sentir angústia psicológica, vergonha, perda de confiança, medo, culpa, depressão e isolamento social. Quanto ao sangramento, este sintoma é descrito como angustiante ${ }^{8}$ e uma fonte de estresse para pacientes, familiares e cuidadores ${ }^{9,3}$.

O sangramento nas feridas tumorais está associado à lesão dos vasos sanguíneos pelas células cancerígenas, podendo ser intensificado pela função plaquetária deficiente e pela estimulação local do fator de crescimento endotelial vascular. Além disso, alguns procedimentos como a troca de curativos, quando não realizadas de forma cautelosa, podem provocar traumas no leito das feridas ou em vasos sanguíneos próximos ao tumor desencadeando os processos hemorrágicos ${ }^{8}$.

As feridas tumorais estão geralmente associadas a prognósticos desfavoráveis, com doença em estágio avançado. Neste contexto, embora abordagens terapêuticas como radioterapia, quimioterapia, terapias hormonais e cirurgia possam promover alívio dos sintomas, essas condutas podem não ser recomendadas a depender do estado clínico do paciente e na maioria das vezes não representam abordagens curativas. Dessa forma, a abordagem mais recomendada no tratamento das feridas tumorais se baseia nos cuidados paliativos. Estes tem como premissa o controle dos sintomas e a garantia de qualidade de vida do paciente ${ }^{10}$.

A forma mais difundida de controle dos sintomas das feridas tumorais é por via tópica. O uso desta via se destaca no controle dos sintomas clássicos dessas feridas, como dor, odor, secreção e sangramento, onde frequentemente é feito o uso off-label de medicamentos e curativos ${ }^{11}$. No que se refere ao sangramento especificamente, a literatura dispõe de uma série de recomendações baseadas em grande parte na aplicação de agentes tópicos, dentre eles se destacam o alginato de cálcio, hemostáticos cirúrgicos e o nitrato de prata ${ }^{9,12}$.
No entanto, observa-se uma escassez de estudos primários com delineamentos metodológicos de alto nível de evidência que demonstrem as melhores condutas e coberturas voltadas ao manejo do sangramento nas feridas tumorais. Destaca-se que a importância da elaboração destes estudos representa não apenas um ganho científico, sobretudo para a prática de enfermagem baseada em evidências, mas reflete também na qualidade de vida dos pacientes que terão seus sintomas controlados, conferindo-lhes maior conforto e segurança. Neste sentido, este estudo tem como objetivo realizar uma revisão integrativa da literatura, focada na identificação e avaliação de estudos primários objetivando o controle tópico do sangramento em feridas tumorais.

\section{MÉTODO}

Trata-se de uma revisão integrativa da literatura a partir da síntese do conhecimento produzido sobre o assunto em diferentes abordagens metodológicas, construída por meio de análise sistemática e ampla dos estudos disponíveis no meio científico $^{13}$. O método da revisão integrativa da literatura é composto de seis etapas: estabelecimento da hipótese ou pergunta da revisão; seleção da amostra a ser revista; categorização e avaliação dos estudos; interpretação dos resultados; e apresentação da revisão ou síntese do conhecimento ${ }^{14}$.

A questão norteadora desta revisão integrativa foi estruturada a partir do sis- 


\section{artigo}

tema PICOD, ou seja, após considerar: a população alvo $(\mathrm{P})$, o interesse da área (I), tipos de intervenção ou cuidados (C), obter resultados e considerar os efeitos a serem alcançados $(\mathrm{O})$ e desenho do estudo (D) resultando na expressão: quais as condutas adotadas para o controle do sangramento em feridas tumorais. Nela, o primeiro elemento da estratégia $(\mathrm{P})$ consiste nos pacientes portadores de feridas tumorais; o segundo (I), as condutas adotadas para o controle tópico do sangramento nas feridas tumorais; o quarto elemento (O) sintetizar um conjunto de evidências a respeito das condutas adotadas para o controle tópico do sangramento em feridas tumorais e o sexto elemento (D) seleção de estudos primários.

A busca na literatura foi realizada entre dezembro de 2020 e Janeiro de 2021 nas seguintes bases de dados: Scopus Info Site
(SCOPUS), Cumulative Index to Nursing \& Allied Health Literature (CINAHL), National Library of Medicine (PubMed) e Web of Science. Foram selecionados descritores a partir do vocabulário internacional utilizado na área da saúde - o Medical Subject Heading (MeSH), criado pela Biblioteca Nacional de Medicina para a literatura indexada no Medline e os descritores em ciências da saúde (DeCS), combinados com operadores booleanos e palavras chaves. Ambos os termos foram combinados de diferentes formas para garantir uma busca ampla (Quadro 1). Houve duas inclusões manuais de artigos que já eram conhecidos pelos pesquisadores.

Os critérios de inclusão para a pré-seleção dos estudos foram: artigos primários, publicados em periódicos e indexados, disponíveis eletronicamente na íntegra, nos idiomas inglês, espanhol ou portu- guês, independentemente do ano de publicação. De acordo com os critérios de exclusão foram excluídos os artigos que não responderam à questão de pesquisa, revisões de literatura, as duplicidades e os que não foram encontrados disponíveis na íntegra. Os artigos achados em duplicidade foram contabilizados na base de dados com maior número de referências.

$\mathrm{O}$ processo de identificação, seleção e inclusão dos estudos foi realizado por dois revisores de forma independente. Divergências entre os revisores foram sanadas com um terceiro revisor. O processo se deu em três etapas. Na primeira etapa foi realizada a retirada dos artigos duplicados; assim, do total de 243 artigos, foram retirados 52. Na segunda etapa, procedeu-se à leitura dos títulos e resumos dos 191 artigos, baseando-se nos critérios de inclusão. Dessa maneira, foram selecionados

\section{Quadro 1. Estratégia de busca e quantitativo de artigos encontrados nas bases de dados. Viçosa, MG, 2021.}

\begin{tabular}{|c|c|c|}
\hline BASES DE DADOS & ESTRATÉGIAS DE BUSCA & ARTIGOS ENCONTRADOS \\
\hline \multirow{2}{*}{ PubMed } & Nursing AND Wounds AND Neoplasms AND Hemorrhage NOT surgery & 64 \\
\cline { 2 - 3 } & Nursing AND "malignant Wounds" AND Hemorrhage AND Symptom \\
management & 5 \\
\hline CINAHL & Hemorrhage AND (Wounds OR Injuries) AND Neoplasms AND Therapeutics & 91 \\
\hline SCOPUS & "Wounds and Injuries" AND bleeding AND neoplasms & 71 \\
\hline Web of Science & TS= bleeding AND TS= "malignant wound" & 10 \\
\hline
\end{tabular}

Figura 1 - Fluxograma de identificação, seleção e inclusão dos estudos da revisão integrativa - Viçosa, MG, Brasil, 2021.

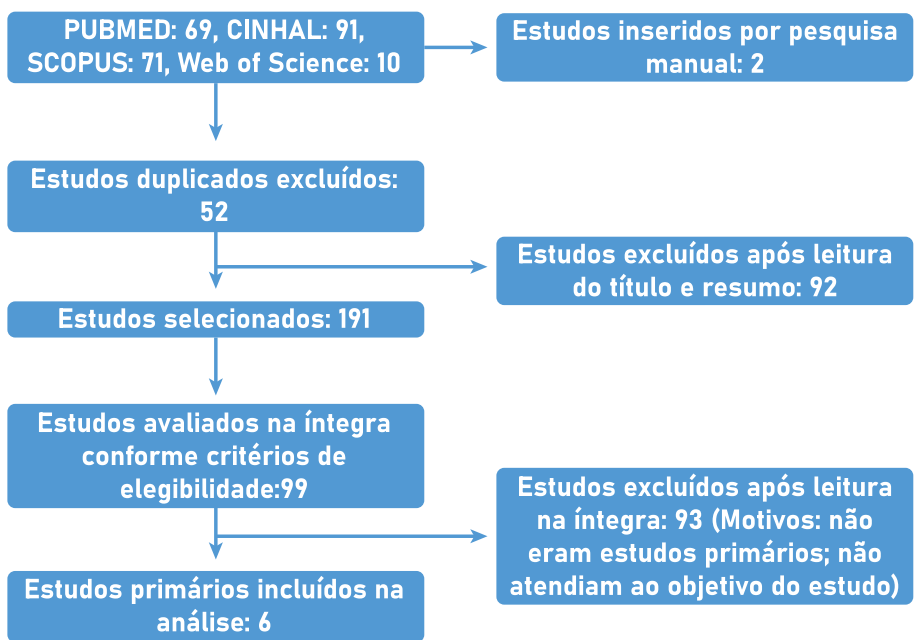

99 artigos. Na terceira etapa realizou-se a leitura na íntegra desses 99 artigos, sendo retirados 93 artigos por não atenderem aos critérios de inclusão e por não responderem à questão norteadora desta revisão, de modo que a amostra final foi constituída por 06 artigos. A Figura 1 ilustra o processo de seleção dos integrativa.

Para o nível de evidência utilizou-se da classificação sugerida por Melnyk e Fineout-Overholt (2005), que classifica os estudos em sete níveis: 1 - evidências provenientes de revisão sistemática ou metanálise de ensaios clínicos aleatorizados controlados ou de diretrizes clínicas baseadas em revisões sistemáticas de ensaios clínicos aleatorizados controlados; 2 - evidências oriundas de pelo menos um ensaio clínico aleatorizado controlado bem de- 
lineado; 3 - evidências obtidas de ensaios clínicos sem aleatorização bem delineados; 4 - evidências que se originaram de estudos de coorte e de caso-controle bem delineados; 5 - evidências originárias de revisão sistemática de estudos descritivos e qualitativos; 6 - evidências derivadas de um único estudo descritivo ou qualitativo; 7 - evidências oriundas de opinião de autoridades e/ou relatório de comitês de especialistas. De acordo com essa classificação, os níveis 1 e 2 são considerados evidências fortes, 3 e 4 moderadas e de 5 a 7 fracas.

\section{RESULTADOS}

A amostra final foi composta por 06 estudos, sendo o mais antigo publicado em 2000 e o mais recente em 2015, dos quais um $(16,7 \%)$ foi publicado em 2000 , um $(16,7 \%)$ em 2010, dois (33,3\%) em 2012, um (16,7\%) em 2014 e um (16,7\%) em 2015. Em relação à origem dos estudos, cinco $(83,3 \%)$ foram publicados no idioma inglês e um (16,7\%) foi publicado no idioma espanhol. Todos os estudos foram publicados em periódicos internacionais. Quanto à localização de realização do estudo, um (16,7\%) foi realizado na França, um $(16,7 \%)$ na Espanha, dois $(33,3 \%)$ no Japão, um (16,7\%) nos EUA e um na Nigéria (16,7\%). Os delineamentos mais frequentes foram série de casos/relato de caso, com cinco estudos (83,3\%), e um estudo de coorte (16,7\%). Portanto, cinco estudos $(83,3 \%)$ apresentaram nível de evidência 6 (fraca) e um (16,7\%), nível de evidência 4 (moderada).

A sinopse dos artigos incluídos neste estudo encontra-se no Quadro 1, contendo a autoria, o título do artigo e o ano de publicação, o nível de evidência, o objetivo, método e as recomendações/conclusões do estudo. Em relação ao nível de evidência, a maioria dos estudos $(83,3 \%)$ apresentou nível 6 que corresponde a evidências derivadas de um único estudo descritivo ou qualitativo, sendo que o método predominante nestes estudos foi estudo de caso/série de casos. Um estudo (16,7\%) apresentou nível de evidência 4, que corresponde a evidências que se originaram de estudos de coorte e de caso-controle bem delineados, sendo um estudo de coorte.

\section{DISCUSSÃO}

A metade dos estudos foi conduzido com pacientes portadores de feridas tumorais ocasionadas por câncer de mama ${ }^{16,17,18}$. Este resultado está em consonância com a literatura que reporta que aproximadamente $50 \%$ de tais feridas estão associadas com este diagnóstico ${ }^{19,20}$. Dois estudos relataram a utilização da pasta de Mosh como tratamento tópico para o sangramento em feridas tumorais. A pasta de Mosh é composta por uma combinação de Cloreto de zinco, água destilada, Pó de zinco e glicerina em concentrações variadas. Foi desenvolvida inicialmente com a finalidade de fixação química de tumor cutâneo durante excisão micrográfica e atualmente, a literatura tem demonstrado que a mesma é eficaz na hemostasia do sangramento de feridas tumorais por meio de aplicação direta ${ }^{17}$.

Embora ambos os estudos tenham relatado desfechos favoráveis na contenção do sangramento, os mesmos apresentaram delineamentos metodológicos pouco robustos, com amostras pouco representativas e sem descrever um controle criterioso em relação ao uso de tratamentos concomitantes à terapêutica estudada e suas possíveis interferências nos resultados, produzindo dessa forma evidências fracas.

Além disso, foi observado que embora os respectivos estudos tenham descrito composições semelhantes no processo de confecção da Pasta de Mosh, não houve uma padronização em relação às concentrações dos componentes. Esta falta de consenso representa um grande problema na utilização desta substância para fins terapêuticos, uma vez que não oferece princípios básicos de segurança, representando riscos à saúde dos pacientes. Este aspecto se torna ainda mais preocupante quando se leva em consideração a alta toxicidade da pasta de Mosh descrita pela literatura ${ }^{21}$.

Limitação metodológica semelhante

\section{Quadro 1 - Apresentação dos estudos incluídos na revisão integrativa, 2021}

\begin{tabular}{|c|c|c|c|c|}
\hline $\begin{array}{l}\text { AUTORES/TITULO } \\
\text { DO ARTIGO/ANO }\end{array}$ & $\begin{array}{l}\text { NIIVEL DE } \\
\text { EVIDÊNCIA }\end{array}$ & $\begin{array}{l}\text { OBJETIVO DO } \\
\text { ESTUDO }\end{array}$ & MÉTODO & $\begin{array}{l}\text { RECOMENDAÇÕES/ } \\
\text { CONCLUSÕES }\end{array}$ \\
\hline $\begin{array}{c}\text { Monleón-Just M, } \\
\text { García YR, López DR, } \\
\text { Borregoa AS, Cres- } \\
\text { poa AA, Jiménez GC. } \\
\text { Cuidados en el dete- } \\
\text { rioro de la integridad } \\
\text { cutánea secundaria } \\
\text { a carcinoma ductal. } \\
2012^{14} \text {. }\end{array}$ & 6 & $\begin{array}{l}\text { Descrever o uso de } \\
\text { um curativo hidro- } \\
\text { celular trilaminar, } \\
\text { com adesivo de } \\
\text { gel macio para o } \\
\text { controle de sin- } \\
\text { tomas (exsudato, } \\
\text { odor, sangramen- } \\
\text { to) e seus efeitos } \\
\text { psicológicos em um } \\
\text { paciente portador } \\
\text { de ferida tumoral } \\
\text { de mama. }\end{array}$ & $\begin{array}{l}\text { Estudo de caso descritivo, realizado com um } \\
\text { paciente do sexo feminino com ferida tumo- } \\
\text { ral em mama. Tempo de acompanhamento } \\
\text { foi de } 6 \text { meses. O sangramento foi avaliado } \\
\text { pela presença ativa de sangue durante as } \\
\text { trocas de curativo. O intervalo de troca dos } \\
\text { curativos foi de } 48 \text { hs durante os primeiros } 3 \\
\text { meses e a cada } 72 \text { hs nos } 3 \text { meses posterio- } \\
\text { res. Condutas para controle do sangramento: } \\
\text { uso da cobertura hidrocelular trilaminar, com } \\
\text { adesivo em gel macio e secagem delicada da } \\
\text { ferida após irrigação. }\end{array}$ & $\begin{array}{l}\text { A cobertura hidrocelular } \\
\text { trilaminar, com adesivo em } \\
\text { gel promoveu uma adesão } \\
\text { delicada ao leito da ferida } \\
\text { evitando traumas durante } \\
\text { as trocas do curativo e } \\
\text { promoveu o controle efe- } \\
\text { tivo do exsudato da ferida } \\
\text { permitindo trocas mais } \\
\text { espaçadas do curativo, } \\
\text { evitando a manipulação } \\
\text { da ferida e consequente- } \\
\text { mente reduzindo o risco de } \\
\text { sangramento. }\end{array}$ \\
\hline
\end{tabular}




\section{artigo}

\begin{tabular}{|c|c|c|c|c|}
\hline $\begin{array}{l}\text { Ladas EJ, Karlik JB, } \\
\text { Rooney D, Taromina } \\
\text { Ndao KDH, Gra- } \\
\text { nowetter L, Kelly } \\
\text { KM. Topical Yunnan } \\
\text { Baiyao administra- } \\
\text { tion as an adjunctive } \\
\text { therapy for bleeding } \\
\text { complications in } \\
\text { adolescents with } \\
\text { advanced câncer. } \\
\text { 2012². }\end{array}$ & 6 & $\begin{array}{c}\text { Descrever o } \\
\text { uso de um pó } \\
\text { medicinal à base } \\
\text { de ervas chinesas } \\
\text { denominado } \\
\text { Yunnan Baiyao } \\
\text { (YNB) para o } \\
\text { controle do } \\
\text { sangramento em } \\
\text { adolescentes com } \\
\text { câncer. }\end{array}$ & $\begin{array}{l}\text { Série de casos com quatro pacientes } \\
\text { com feridas tumorais decorrentes de: } \\
\text { sarcoma sinovial em mandíbula e cavidade } \\
\text { oral; Sarcoma sinovial axilar direito e } \\
\text { parede torácica; Linfoma de Burkitt e } \\
\text { rabdomiossarcoma alveolar recidivante. } \\
\text { Protocolo de utilização do YNB: Paciente } \\
\text { 1: aplicação tópica de cápsulas de YNB, } \\
\text { esmagadas e mergulhadas em uma } \\
\text { esponja estéril. Não foi mencionado tempo } \\
\text { específico de uso. Paciente 2: Aplicação } \\
\text { de YNB em uma esponja diretamente } \\
\text { na superfície em sangramento quantas } \\
\text { vezes forem necessárias para sessar o } \\
\text { mesmo. Paciente 3: aplicação tópica do YNB } \\
\text { associado à vaselina. Não foi mencionada } \\
\text { a frequência de aplicação. Foi orientado } \\
\text { como uso profilático a aplicação da erva com } \\
\text { um cotonete topicamente duas vezes ao } \\
\text { dia, por } 1 \text { semana. Paciente 4: YNB tópico } \\
\text { associado a comprimidos de vaselina e } \\
\text { ácido aminocapróico na vigência de episódio } \\
\text { hemorrágico. }\end{array}$ & $\begin{array}{l}\text { Todos os pacientes do } \\
\text { estudo obtiveram controle } \\
\text { do sangramento. No } \\
\text { entanto, não foi possível } \\
\text { avaliar a contribuição exata } \\
\text { do YNB para hemostasia } \\
\text { das feridas tumorais, visto } \\
\text { que o pó foi utilizado como } \\
\text { terapia adjuvante. }\end{array}$ \\
\hline $\begin{array}{l}\text { Kakimoto M, Tokita } \\
\text { H, Okamura T, } \\
\text { Yoshino Koji. A } \\
\text { Chemical Hemostatic } \\
\text { Technique for Blee- } \\
\text { ding from Malignant } \\
\text { Wounds. 2010 }\end{array}$ & 6 & $\begin{array}{l}\text { Realizar tratamento } \\
\text { químico hemos- } \\
\text { tático com pasta } \\
\text { de cloreto de zinco } \\
\text { (Pasta de Mohs), } \\
\text { em feridas tumo- } \\
\text { rais de mama com } \\
\text { sangramento. }\end{array}$ & $\begin{array}{l}\text { Série de casos com cinco pacientes com } \\
\text { câncer de mama. Foi realizado tratamento } \\
\text { químico hemostático com pasta de Mohs. } \\
\text { Aplicação tópica da pasta de Mohs na } \\
\text { superfície tumoral com o auxílio de astes } \\
\text { de madeira ou aplicação da pasta na gaze e } \\
\text { compressão desta diretamente nos locais de } \\
\text { sangramento ativos no tumor. Foi menciona- } \\
\text { do um tempo de aplicação de } 5 \text { a } 10 \text { min para } \\
\text { sessar o sangramento. }\end{array}$ & $\begin{array}{l}\text { O tratamento hemos- } \\
\text { tático foi bem-sucedido } \\
\text { na primeira aplicação em } \\
\text { todos os cinco pacientes. } 0 \\
\text { controle do sangramento } \\
\text { foi mantido de } 3 \text { semanas a } \\
\text { mais de } 3 \text { meses. }\end{array}$ \\
\hline $\begin{array}{l}\text { Fromantin I, Watson } \\
\text { S, Baffie A, Rivat } \\
\text { A, Falcou M-C, } \\
\text { Kriegel I, Ingenior } \\
\text { YR. A prospective, } \\
\text { descriptive cohort } \\
\text { study of malignant } \\
\text { wound characteris- } \\
\text { tics and wound care } \\
\text { strategies in patients } \\
\text { with breast câncer. } \\
2014^{16} \text {. }\end{array}$ & 4 & $\begin{array}{l}\text { Avaliar o uso de } \\
\text { terapias tópicas e } \\
\text { características de } \\
\text { feridas tumorais. }\end{array}$ & $\begin{array}{l}\text { Coorte prospectiva durante } 20 \text { meses (maio } \\
\text { de } 2010 \text { a janeiro de 2011), conduzido com } \\
32 \text { pacientes com câncer de mama. Foram } \\
\text { elencados critérios de inclusão e exclusão. } \\
\text { Foram feitas avaliações em um período de } 42 \\
\text { dias, com uma avaliação a cada } 21 \text { dias (dia 0, } \\
\text { dia } 21 \text { e dia 42). Foram investigados infecção, } \\
\text { dor, odor, exsudato e sangramento. O san- } \\
\text { gramento foi considerado espontâneo quan- } \\
\text { do ocorreu entre duas trocas de curativo, ou } \\
\text { induzida quando ocorreu durante a troca de } \\
\text { curativo. Curativos: alginatos, hemostáticos e } \\
\text { adrenalina local para sangramento espontâ- } \\
\text { neo e aplicação de um curativo não aderente } \\
\text { do tipo interface ou impregnado de silicone } \\
\text { como rotina. }\end{array}$ & $\begin{array}{l}\text { O sangramento foi contro- } \\
\text { lado pelos cuidados locais } \\
\text { com a ferida. O número } \\
\text { de pacientes incluídos no } \\
\text { estudo foi muito peque- } \\
\text { no para tirar quaisquer } \\
\text { conclusões robustas sobre } \\
\text { as diretrizes de cuidados. } \\
\text { O período de avaliação do } \\
\text { estudo foi considerado } \\
\text { relativamente curto para } \\
\text { avaliar a evolução de uma } \\
\text { ferida crônica. }\end{array}$ \\
\hline
\end{tabular}




\begin{tabular}{|c|c|c|c|c|}
\hline $\begin{array}{c}\text { Yamano T, Kobayashi } \\
\text { M, Yoshimura M, } \\
\text { Tsukamoto K, Noda } \\
\text { M, Matsubara N, Na- } \\
\text { gita A, Naohiro T. The } \\
\text { usefulness of Mohs' } \\
\text { paste for the treat- } \\
\text { ment of a malignant } \\
\text { wound caused by a } \\
\text { giant intra-abdomi- } \\
\text { nal desmoid tumor. } \\
2015^{19} \text {. }\end{array}$ & 6 & $\begin{array}{l}\text { Relatar a utilização } \\
\text { da Pasta de Mohs } \\
\text { no controle de } \\
\text { sintomas de uma } \\
\text { ferida tumoral origi- } \\
\text { nada a partir de um } \\
\text { tumor desmóide } \\
\text { (TD). }\end{array}$ & $\begin{array}{l}\text { Relato de caso. Aplicação de pasta de Mohs } \\
\text { em ferida de um tumor desmóide intra-ab- } \\
\text { dominal. Foi feita uma preparação à base } \\
\text { de } 100 \mathrm{~g} \text { de cloreto de zinco, } 50 \mathrm{~g} \text { de zinco } \\
\text { em pó, } 10 \mathrm{~mL} \text { de glicerol em } 50 \mathrm{~mL} \text { de água } \\
\text { destilada e aplicada em gaze. A mesma foi } \\
\text { colocada na superfície do tumor. A gaze foi } \\
\text { trocada a cada dois dias até que o tumor } \\
\text { estivesse necrótico. Duração de } 10 \text { meses. }\end{array}$ & $\begin{array}{l}\text { A Pasta de Mohs se mons- } \\
\text { trou efetiva no controle } \\
\text { do sangramento da ferida } \\
\text { tumoral oriunda do tumor } \\
\text { desmóide. O estudo ressal- } \\
\text { tou uma preocupação com } \\
\text { danos aos órgãos adja- } \\
\text { centes ao tumor visto que } \\
\text { a pasta de Mohs é muito } \\
\text { tóxica. }\end{array}$ \\
\hline $\begin{array}{l}\text { Adebamowo CA. } \\
\text { Topical Formalin } \\
\text { for Management of } \\
\text { Bleeding Malignant } \\
\text { Ulcers. } 2000^{20} \text {. }\end{array}$ & 6 & $\begin{array}{l}\text { Avaliar o uso de } \\
\text { Formalina tópica } \\
\text { em um grupo de } \\
\text { pacientes com } \\
\text { sangramento em } \\
\text { feridas tumorais. }\end{array}$ & $\begin{array}{l}\text { Série de casos com } 22 \text { pacientes com feridas } \\
\text { tumorais. O controle do sangramento foi } \\
\text { feito por meio de curativos. Foi realizada apli- } \\
\text { cação tópica de gaze de algodão embebida } \\
\text { em Formalina } 10 \% \text { na superfície do tumor. A } \\
\text { gaze permanecia no leito da ferida até cessar } \\
\text { o sangramento ou por tempo máximo de } 3 \\
\text { minutos. O procedimento foi repetido em } \\
\text { intervalos de } 12 \text { horas caso o sangramento } \\
\text { persistisse. }\end{array}$ & $\begin{array}{l}\text { A Formalina tópica foi } \\
\text { efetiva no controle do } \\
\text { sangramento em apenas } \\
\text { uma aplicação em } 17 \text { pa- } \\
\text { cientes (77,3\%); na segunda } \\
\text { aplicação em dois pacientes } \\
(9,1 \%) \text { e seis a oito vezes } \\
\text { em dois pacientes }(9,1 \%) \text {, } \\
\text { onde não foi alcançada a } \\
\text { hemostasia. Os pacientes } \\
\text { relataram uma sensação } \\
\text { "apimentada" durante a } \\
\text { aplicação. }\end{array}$ \\
\hline
\end{tabular}

foi identificada no estudo de Adebamowo $(2000)^{22}$, que buscou avaliar o uso de Formalina tópica em uma série de casos. $\mathrm{O}$ uso da formalina foi inicialmente reportado visando o controle do sangramento da cistite hemorrágica ${ }^{23}$, e posteriormente foi usada para tratamento de sangramento oriundo de retite por radiação ${ }^{24,25}$, além disso é um fixador de tecidos, conservante, desinfetante e agente de embalsamamento.

O estudo demonstrou que apenas uma aplicação da Formalina tópica foi efetiva no controle do sangramento em $77,3 \%$ dos pacientes, além disso houve certa padronização do procedimento de aplicação da mesma e tempo de duração, porém os autores ressaltaram que o volume de absorção da substância no organismo não é conhecido e que embora a formalina tenha sido administrada no estudo em uma concentração média de $10 \mathrm{ml}$, considerada segura pelos autores, outros estudos não corroboram essa informação.

Outro estudo ${ }^{17}$ desta amostra descreveu o uso de um pó medicinal à base de ervas chinesas denominado Yunnan Baiyao (YNB) para o controle do sangramento em uma série de casos composta por cinco pacientes com feridas tumorais derivadas de sarcoma sinovial recorrente em mandíbula esquerda e cavidade oral, sarcoma sinovial axilar direito e parede torácica, Linfoma de Burkitt e rabdomiossarcoma alveolar recidivante. O Yunnan Baiyao (YNB) se caracteriza por um Pó medicinal à base de ervas chinesas (Panax notoginseng, Ajuga forrestii Diels, Dioscoreae Parviflora Ting, Herba Inulae Cappae, Herba Geranii e Herba Erodii, Rhizoma Dioscoreae e Rhizoma Dioscoreae Nipponicae e cerca de $40 \%$ de extrato de raiz seca de P. notoginseng) formulado incialmente em 1902.

A respeito da eficácia do YNB como hemostático, um pequeno ensaio duplo-cego controlado por placebo foi realizado a fim de investigar as propriedades hemostáticas do YNB na prevenção da perda sanguínea em adultos submetidos à cirurgia ortopédica maxilar. Para tanto, o YNB foi administrado por via oral por 3 dias antes da cirurgia. O estudo identificou uma perda sanguínea significativamente menor no grupo tratado em relação ao grupo controle, além disso não foram identificados eventos tromboembólicos ou outros efei- tos colaterais ${ }^{26}$. Outros dois estudos chineses relataram que a administração oral de YNB diminuiu o sangramento intraoperatório durante um procedimento de laminoplastia e durante a ressecção transuretral de próstata, demonstrando taxas de efeitos colaterais semelhantes entre os grupos tratados e controle ${ }^{27}$.

Embora os estudos supracitados tenham delineamentos metodológicos mais consistentes com randomização e duplo-cegamento, os mesmos foram realizados em pacientes cirúrgicos e as mesmas evidências não podem ser inferidas para o sangramento derivado de feridas tumorais, visto que a fisiopatologia do sangramento nessas feridas é específica e associada ao processo de oncogênese.

Embora o estudo de Ladas et al $(2012)^{28}$ tenha se proposto a fornecer essas evidências científicas para o contexto oncológico e tenha apresentado um desfecho favorável quanto ao controle do sangramento, o mesmo demonstrou baixa evidência. Suas maiores fragilidades foram identificadas em relação à falta de padronização quanto ao protocolo de aplicação do YNB, a apresentação variada da substância e o uso con- 
comitante de outros tratamentos tópicos para o sangramento como o ácido aminocapróico o que se configurou como grande fator de confundimento para a investigação dos efeitos terapêuticos da substância.

Dois estudos da amostra não investigaram uma substância tópica específica para o controle do sangramento, mas abordaram como estratégias terapêuticas o uso de coberturas e técnicas de curativo específicas ao manejo das feridas oncológicas friáveis. O estudo de Monleón-Just et al $(2012)^{16}$, buscou descrever o uso de um curativo hidrocelular trilaminar, com adesivo de gel macio em um paciente portador de ferida tumoral em mama visando o controle do exsudato, do odor e do sangramento, além de avaliar os efeitos psicológicos do tratamento.

A cobertura avaliada promoveu uma adesão delicada ao leito da ferida evitando traumas durante as trocas do curativo e realizou o controle efetivo do exsudato da ferida permitindo trocas mais espaçadas do curativo, evitando a manipulação da ferida e consequentemente reduzindo o risco de sangramento. Como fatores limitantes, o artigo apresentou uma baixa evidência devido ao seu tamanho amostral insatisfatório e delineamento experimental limitado.

Resultado semelhante foi identificado no estudo de Fromantin et al (2014), o único estudo da amostra desta revisão que apresentou evidência moderada. $\mathrm{O}$ estudo avaliou o uso de terapias tópicas no controle de sintomas de feridas tumorais, dentre eles, o sangramento. Para tanto, foi feito um estudo de coorte prospectivo durante 20 meses (maio de 2010 a janeiro de 2011), conduzido com 32 pacientes com ferida tumoral resultante de câncer de mama. $\mathrm{O}$ estudo apresentou um delineamento metodológico mais robusto, descrevendo os critérios de inclusão e exclusão, descrição detalhada dos materiais e procedimentos realizados e definição dos critérios de avaliação do controle dos sintomas.

As principais lacunas evidenciadas no estudo foram com relação a um número reduzido da amostra de pacientes, o que não permite estabelecer conclusões robustas sobre as diretrizes de cuidados. Além disso,
A cobertura

avaliada promoveu

uma adesão

delicada ao leito

da ferida evitando

traumas durante as

trocas do curativo

e realizou o

controle efetivo do

exsudato da ferida

permitindo trocas

mais espaçadas do

curativo, evitando

a manipulação

da ferida e

consequentemente

reduzindo o

risco de

sangramento. o período de avaliação do estudo foi de 42 dias, período considerado relativamente curto para avaliar a evolução de uma ferida crônica. Ambos os estudos evidenciaram respectivamente a importância da utilização de técnica adequada pelos profissionais durante a realização do curativo em feridas tumorais e a efetividade do controle do sangramento dessas feridas por meio da utilização de curativos à base de alginatos, hemostáticos e adrenalina em caso de sangramento local espontâneo e aplicação de curativo não aderente do tipo interface ou impregnado de silicone como rotina.

É importante ressaltar que a abordagem tópica e mais conservadora do sangramento de feridas tumorais realizado por meio de curativos se mostra preferível para grande parte desses pacientes, sobretudo porque os mesmos não apresentam condições clínicas satisfatórias para serem submetidos a procedimentos anti-hemorrágicos mais agressivos como ressecamento cirúrgico ou radioterapia anti-hemorrágica, além desses procedimentos mais conservadores proporcionarem um maior conforto e melhor qualidade de vida a esses pacientes. É sabido que em alguns casos, a abordagem tópica não é suficiente no controle do sangramento, sobretudo em caso de rompimento de grandes vasos que ocasionam hemorragias profusas, nestes casos, procedimentos como embolização ou sutura do vaso são altamente recomendados ${ }^{6,8}$.

\section{CONCLUSÃO}

O presente estudo buscou reunir as evidências disponíveis na literatura a respeito das melhores práticas e recomendações associadas ao controle do sangramento em feridas tumorais. Embora as evidências aqui apresentadas não sejam altas, os estudos descreveram uma série de substâncias, coberturas e procedimentos que tem sido utilizadas pelos profissionais no manejo prático deste sintoma apresentando resultados satisfatórios. Mesmo não se configurando como evidências robustas e não sendo possível uma generalização dos resultados, o conhecimento disponível até o 
momento já representa um avanço significativo em termos de promoção de qualidade de vida para os pacientes e seus familiares e este é o objetivo central dos cuidados paliativos. Neste sentido, recomenda-se que estudos posteriores com melhor delineamento metodológico sejam conduzidos a fim de produzir evidências mais concretas a respeito dos medicamentos tópicos aqui apresentados, permitindo que sejam utilizados com maior segurança e em um maior número de pacientes.

\section{REFERÊNCIAS}

1. Tilley C, Lipson J, Ramos M. Palliative wound care for malignant fungating wounds: holistic considerations at end of life. Nurs Clin North Am. 2016;51(3):513-31.

2. Instituto Nacional de Câncer (Inca). Tratamento e controle de feridas tumorais e úlceras por pressão no câncer avançado. Rio de Janeiro: Inca; 2009.

3. Probst S, Arber A, Faithfull S. Coping with an exulcerated breast carcinoma: an interpretative phenomenological study. J Wound Care. 2013; 22(7):352-360.

4. Tandler S, Stephen-Haynes J. Fungating wounds: management and treatment options. British Journal of Nursing 2017; 26(12): S6-S14.

5. Santos CMC, Pimenta CAM, Nobre MRC. A systematic review of topical treatments to control the odor of malignant fungating wounds. J Pain Symptom Manage. 2010; 39(6):1065-76.

6. Seaman S, Bates-Jensen BM. Skin disorders. Malignant wounds, fistulas, and stomas. In: Ferrell BR, Coyle N, Paice JÁ (eds). Oxford Textbook of Palliative Nursing (4th edn). Oxford University Press, 2015.

7. Lund-Nielsen B. Malignant wounds in patients with advanced stage cancer-quantitative and qualitative findings. Faculty of Health Sciences, University of Copenhagem, 2011.

8. European Oncology Nursing Society. Recommendations for the Care of Patients with Malignant Fungating Wounds. London, UK: EONS. 2015.

9. Alexander SJ. An intense and unforgettable experience: the lived experience of malignant wounds from the perspectives of patients, caregivers and nurses. Int Wound I 2010; 7(6):456-465.

10. Tandler S, Stephen-Haynes J. Fungating wounds: management and treatment options. British Journal of Nursing. 2017; 26(12): S6-514.

11. Firmino F, Villela-Castro DL, Santos J, Santos VLCG. Topical Management of Bleeding From Malignant Wounds Caused by Breast Cancer: A Systematic Review. Journal of Pain and Symptom Management. 2020;0(0): 1-9.

12. Piggin C, Jones V. Malignant fungating wounds: an analysis of the lived experience. J Wound Care 2009; 18(2):57-64.

13. Pereira MG. Artigos científicos: como redigir, publicar e avaliar. Rio de Janeiro: Guanabara Koogan; 2011.

14. Mendes KDS, Silveira RCCP, Galvão CM. Integrative literature review: a research method to incorporate evidence in health care and nursing]. Texto Contexto Enferm. 2008;17(4):758-64.

15. Melnyk BM, Fineout-Overholt E. Evidence-based practice in nursing \& healthcare: a guide to best practice. Philadelphia: Lippincot; 2005. Making the case for evidence-based practice;

\section{p. 3-24.}

16. Monleon-Just M, Garcia YR, Ruiz-Lopez D, et al. Care in the deterioration of skin integrity due to ductal carcinoma. Palliat Med. 2012;19:155e159.

17. Kakimoto M, Tokita H, Okamura T, Yoshino K. A chemical hemostatic technique for bleeding from malignant wounds. J Palliat Med. 2010;13:11e13.

18. Fromantin I, Watson S, Baffie A, et al. A prospective, descriptive cohort study of malignant wound characteristics and wound care strategies in patients with breast cancer. Ostomy Wound Manage. 2014;60:38e48.

19. Maida V, Ennis M, Kuziemsky C, Trozzolo L: Symptoms associated with malignant wounds: A prospective case series. J Pain Symptom Manage. 2009;37:206-211.

20. Schulz V, Triska OH, Tonkin K: Malignant wounds: Caregiver-determined clinical problems. J Pain Symptom Manage. 2002;24:572-577.

21. Yamano $T$, Kobayashi M, Yoshimura M, Tsukamoto K, Noda M, Matsubara N, Nagita A, Naohiro T. The usefulness of Mohs' paste for the treatment of a malignant wound caused by a giant intra-abdominal desmoid tumor. Int Canc Conf J. 2015;1:1-4.

22. Adebamowo AC. Topical formalin for management of bleeding malignant ulcers. J Palliat Med. 2000;24:518e520.

23. Brown RB. A method of management of inoperable carcinoma of the bladder. Med. J. Aust. 1969;1:23.

24. Mathai V. Seow-Choen, F.: Endo-luminal formalin therapy for haemorrhagic radiation proctitis. Br. J. Surg. 1995;82:190.

25. Roche B, Chautems R, Marti MC. Application of formaldehyde for the treatment of haemorrhagic radiation induced proctitis. World J. Surg. 1996; 20:1095.

26. Tang ZL, Wang X, Yi B, Li ZL, Liang C, Wang XX. Effects of the preoperative administration of YNB capsules on intraoperative blood loss in bimaxillary orthognathic surgery: a prospective, randomized, double-blind, placebo-controlled study. Int J Oral Maxillofac Surg. 2009;38(3):261-266.

27. Pan SF, Sun Y, Li F, Li GH, Jin DD, Zhang ZM, Luo ZJ, Ye ZX, Hu $J H$, Qiu GX. Effects of YNB on peri-operative bleeding of patients undergoing cervical open-door laminoplasty: a multicenter randomized double-blind placebo-control trial. Zhonghua Yi Xue Za Zhi. 2006; 86(27):1888-1890.

28. Ladas EJ, Karlik JB, Rooney D, Taromina K, Ndao DH, Granowetter L, Kelly KM. Topical Yunnan Baiyao administration as an adjunctive therapy for bleeding complications in adolescents with advanced câncer. Support Care Cancer. 2012;20:33793383 